\title{
An Efficient and Leightweight Illumination model for Planetary Bodies including Direct and Diffuse Radiation
}

\author{
Marco Scharringhausen \\ German Aerospace Center \\ Institute of Space Systems \\ Bremen, Germany
}

\author{
Lars Witte \\ German Aerospace Center \\ Institute of Space Systems \\ Bremen, Germany
}

12. April 2018

\section{Zusammenfassung}

We present a numerical illumination model to calculate direct as well as diffuse or Hapke scattered radiation scenarios on arbitrary planetary surfaces. This includes small body surfaces such as main belt asteroids as well as e.g. the lunar surface. The model is based on the raytracing method. This method is not restricted to spherical or ellipsiodal shapes but digital terrain data of arbitrary spatial resolution can be fed into the model. Solar radiation is the source of direct radiation, wavelength-dependent effects (e.g. albedo) can be accounted for. Mutual illumination of individual bodies in implemented (e.g. in binary or multiple systems) as well as self-illumination (e.g. crater floors by crater walls) by diffuse or Hapke radiation. The model is validated by statistical methods. A $\chi^{2}$ test is undertaken to compare simnulated images with DAWN images acquired during the survey phase at small body 4 Vesta.

\section{Introduction}

Reliable prediction or at least estimation of illumination conditions on the surface of planetary bides (e.g. the Earth moon) or small bodies like asteroids or comet cores is essential for mission planning purposes.

In general, mission planning for any vehicle on a strategic (before start of the mission) and/or tactical level (during the mission) requires knowledge about environmental conditions at the destination. From a general point of view and depending on the vehicle(s) involved, this might include atmospheric properties such as density, wind speed as well as surface properties such as roughness, slope, gravity and illumination. This applies not only to surface elements but also to planetary landers.

Firstly, thermal design of any lander space craft relies on a reliable information about the environment (e.g. [2]). As solar radiation is in general the major source of energy on the surface of a small, atmosphereless body in space, a simulation suite for calculation of the surface radiative intensity delivers boundary conditions for any thermal model of the surface.

The same applies to the design of a mobile surface element powered by solar cells, e.g. a rover. For rovers, additional aspects come into play, i.e. not only the thermal and power budget are affected by illumination conditions but also the path and trajectory planning of the rover itself ([14], [7]). Being able to reliably predict illumination at any time and at any point on the surface of the small or planetary body is an essential part of the cost function in any optimization process. During the mission, the rover's mission plan may involve travel to several distinct sites, interleaving periods of dedicated science data collection with periods of traversal and opportunistic science. To repower internal batteries, it might be beneficial to pause for a period of time to exploit time slots of maximal illumination. To plan all this in advance at least on a tactical, if not on a strategic level, an illumination simulation is 


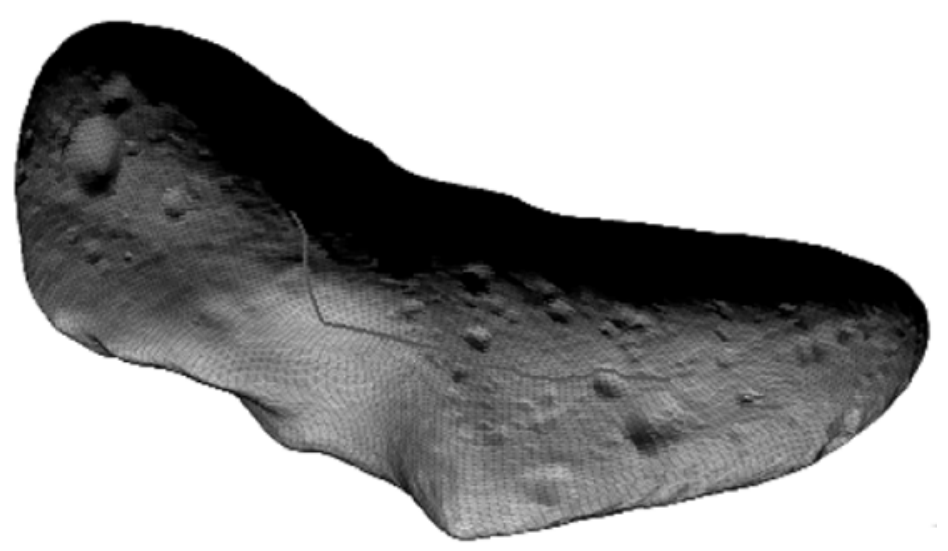

Abbildung 1: Optimal path of some generic rover on the surface of (433) Eros. The cost function involves illumination, surface soughness ans slope to equal weights. The light source (point) is located at the bottom letf hand side corner, shading indicates illumination.

crucial. Also, it may be beneficial to have the illumination simulation as leightweight as possible to have it run onboard the rover.

Also, fundamental research questions rely on high-quality estimates of the radiation and thermal environment on the body surface. This applies e.g. to the formation of cometary tails and halos but also to the varying ice content of asteroids.

\section{Illumination Analysis: State of the Art}

To date, a number of illumination simulation tools and suites are accessible for the scientific community.

The PANGU (Planet and Asteroid Natural Scene Generation Utility) tool [15] for simulation and visualization of planetary surfaces has been designed to support the development of landers that use computer vision to navigate towards the surface and to avoid any obstacles near the landing site. Its primary data product are artificial camera images. It can be used to generate an artificial surface representative of cratered planets. Crater and boulder distributions can be prescribed or parametrized. Camera position and line-of-sight on or above the planetary surface can be chosen arbitrarily. Note that PANGU was originally developed to simulate the surface of heavily cratered planetary bodies like the Moon and Mercury ([15], [16]). It is thus not as generalistic as it may be and needs to be extended.

PANGU includes the option to simulate surfaces with atmospheres as e.g. Mars. PANGU builds a cratered planet surface model by simulating impact cratering on an initial terrain model. Craters are put on the terrain either manually or randomly according to a user defined crater size-density distribution. Those crater models combine idealised impact crater models with fractal techniques to produce a "realisticäppearance to the craters. However, it has been found that explicit crater or boulder distributions given by external data sources may not be treated correctly [REFERENZ ESA LL].

The SurRender is a software toolkit for the scientific-level generation of space scene images. It is propretiary software of Airbus Defence and Space. It primary data product are camera images. The image generation method is raytracing, as is the case for our tool. Diffraction by atmospheres as well as multiple scattered light (both by atmosphere and ground) can be included in the images. Also, arbitrary bidrectional reflectance functions (BDRF) such as Lambert, Hapke (for regolith-covered moons and asteroids), Phong and Oren-Nayar (for Jovian moons) can be chosen by the user. Additionally, a sensor model can be delivered by the user, including geometry, optics, motion, sensor, noise and electronics. 


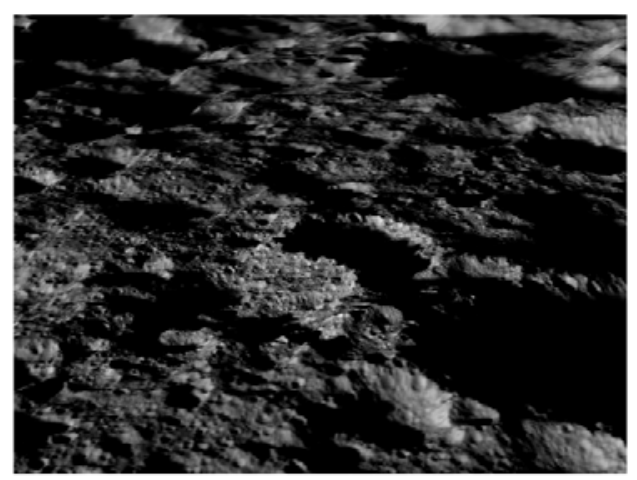

(a) Cratered lunar surface rendered by PANGU [15].
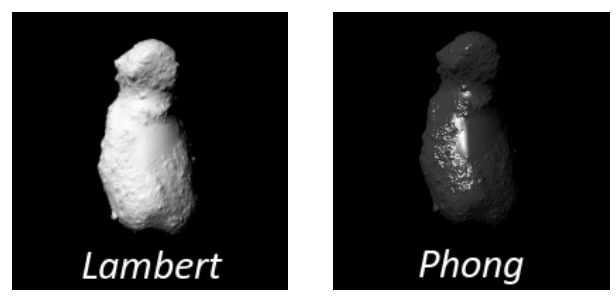

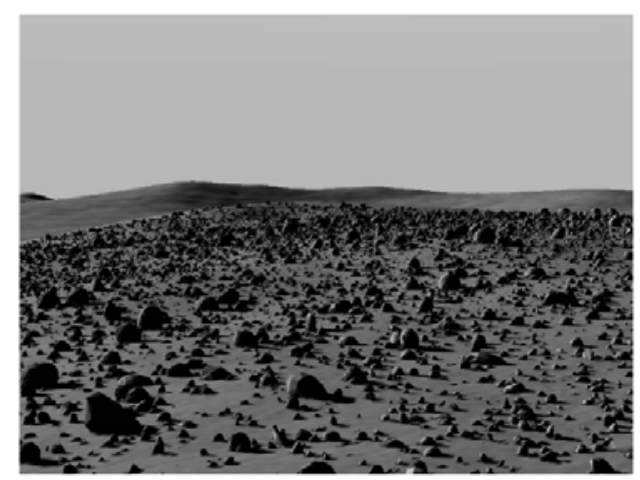

(b) Martian surface with rocks and boulders rendered by PANGU [15].
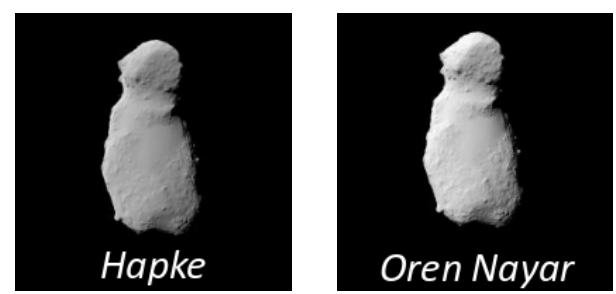

Abbildung 3: Bidirectional reflectance functions implemented in SurRender.

Also, SurRender is able to include textures on a planetary scale. These textures can be user-provided by external data as well as internally calculated by SurRender. The latter case yields then procedural textures. Textures can be mapped to digital terrain models.

SurRender is optimized for space scenarios, i.e. large distances between object and sensor. This is in line with the advantages inherent to raytracing, since the raytracing method is well suited to render sparse scenes, as is the case for empty space and large distances between object and camera.

\section{Illumination Analysis: The SLIM model}

The Space-Scene Leightweight Illumination Model (SLIM) is designed to be leightweight and efficiently deliver illumination intensities on the surface ot a planetary body. It primary data product is thus not a camera image but the physical radiance or irradiance on the surface of any body. The camera images generated for this study are used for validation against real remote sensing data (e.g. DAWN mission). This method is preferred since large scale illumination measurements are not available yet for any atmosphereless planetary body in the solar system. Point measurements of illumination have been recorded from various lander missions, however this has never been done on a near-global scale.

SLIM offers the option to do inversion, i.e. extraction of optical surface properties - Hapke parameters or albedo - from camera images. This inversion can be done not only for diskintegrated data sets but surface parameters can be calculated but also on scales of a couple of triangles.

Illumination calculated by SLIM can be fed directly into a thermal model or any vehicle power model, since the data is exported in general ASCII format. The SLIM model is versatile since surfaces can be handed to the model as triangulations given in PLY, STL or OBJ format.

Surfaces do not need to be closed, can thus be small patches of any body surface. Bodies of arbitrary size (asteroids, moons, planets) can be handled with the same code and user 
interface. Crater and boulder distributions cannot be parametrized but need to be explicitly provided (as part of the triangulated surface) by the user. It should be noted, however, that the planetary surface itself and the bould/rocks on top of it can be input in separate files, there is no need to merge the two teiangulations beforehand. Surfaces can be of arbitrary shape, i.e. do not need to be convex but can have edges, bulges, juts and overhangs, consider e.g. (25143) Itokawa (fig. 4b)

Surfaces can have arbitrary spatial resolution, triangles of almost (up to numerical limits) sizes can be handled simultanously in the same data set.

\section{Small body data model}

The small body data used in this study come as point coordinates of surface points in $\mathbb{R}^{3}$ alongside with a triangulation. No points or triangles are located in the interior of the bodies. Number of triangles patches can vary from a few hundred up to a few million, depending on data availability and requirements on the accuracy of the illumination analysis.

Data can be gained from remote sensing methods such as earth-based radar observations (as is the case e.g. for Phobos) as well as from close-encounter orbiter data (LIDAR etc.), as is the case e.g. for (25143) Itokawa, see figure 4.

\section{$5 \quad$ Ephemerides}

All relevant ephemerides data of body, spacecraft and sun are calculatced by the SPICE toolkit vN0066. This yields the position of the S/C as well as the line-to-the-sun (LTS) and the line-of-sight (LOS) of the camera in the body-fixed coordinate system.

For the case study presented later, imagery data 4 Vesta as acquired by the Dawn mission have been utilized and ephemerides data are given in the Dawn-Claudia coordinate system for Vesta.

\section{Radiation}

The sun is considered the only source of direct radiation. Bodies are assumed to be able to illuminate each other (e.g. in binary systems) or themselves (e.g. crater floors by crater walls) by diffuse radiation. Lambertian diffuse scattering can be accounted for up to arbitrary orders of scattering by using the method of radiosity ([6], [1], [19], [10]). For diffuse scattering following a non-lambertian reflectance function, only the first order of scattering is accounted for.

All quantities are considered to be independent of wavelength. This is an approximation to reality, e.g. albedo (see fig. (6.2)) is wavelength dependent. For practical purposes, making quantities wavelength independent can be accomplished by averaging over a certain wavelength range. Alternatively, the illumination algorithm presented here can be used for single wavelengths or wavelength intervals and the results superpositioned afterwards. That is, "colourïmages can be rendered by individually rendering monochromatic images using different values for relevant physical quantities such as albedo, Hapke parameters etc. The corresponding set of monochromatic images can then be superpositioned to yield an polychromatic image. For the calculation of surface illumination, intensities of radiation can be calculated for a predefined set of wavelengths and then added to yield the multispectral illumination, see fig. 5 and 6 . 


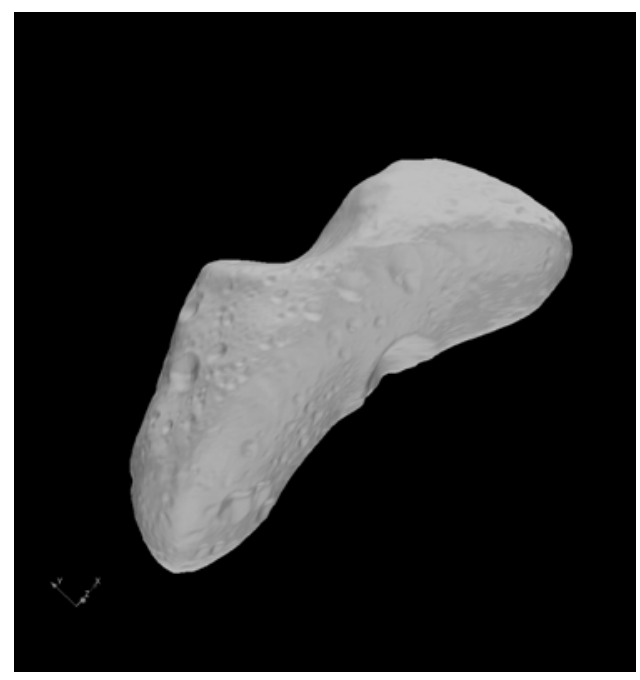

(a) Asteroid (433) ERos. 196608 faces, average triangle area $5771 \mathrm{~m}^{2}$, average edge length approx. 115 metres [4].

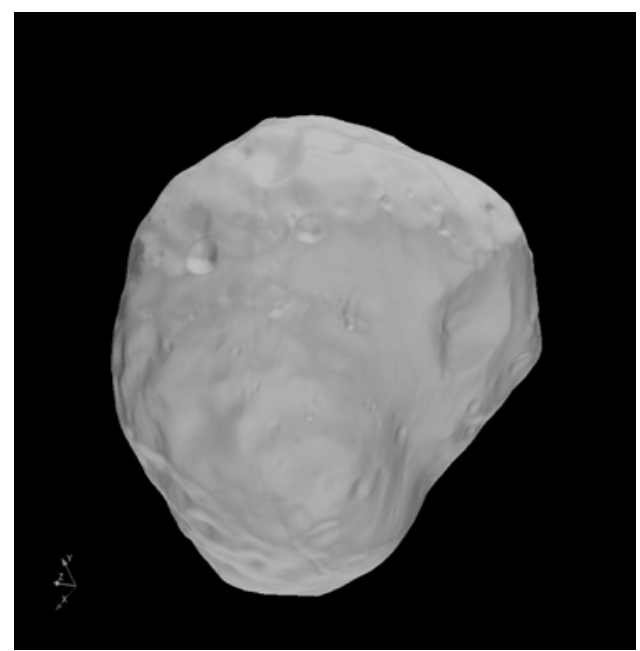

(c) Martian satellite Рновоs, 49152 faces, average triangle area $33500 \mathrm{~m}^{2}$, average edge length approx. 278 metres [5].

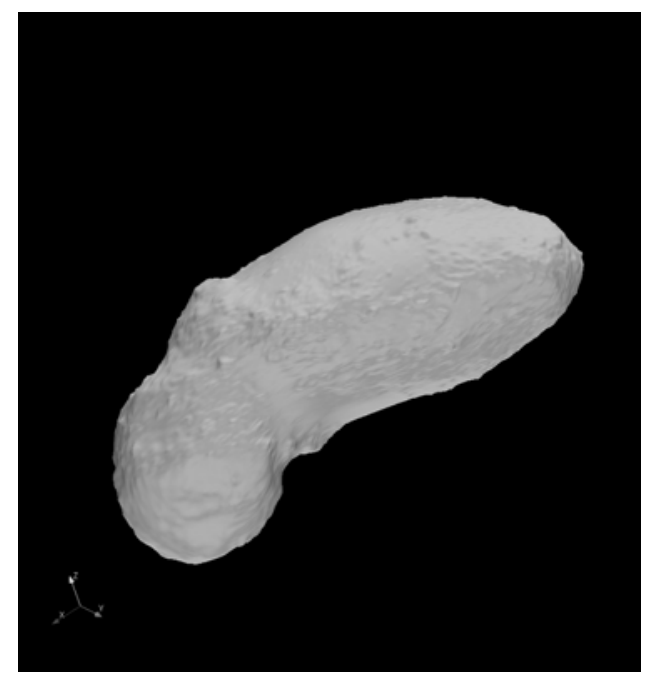

(b) Asteroid (25143) ITOKAWA, 196608 faces, average triangle area $2 \mathrm{~m}^{2}$, average edge length approx. 2 metres [3].

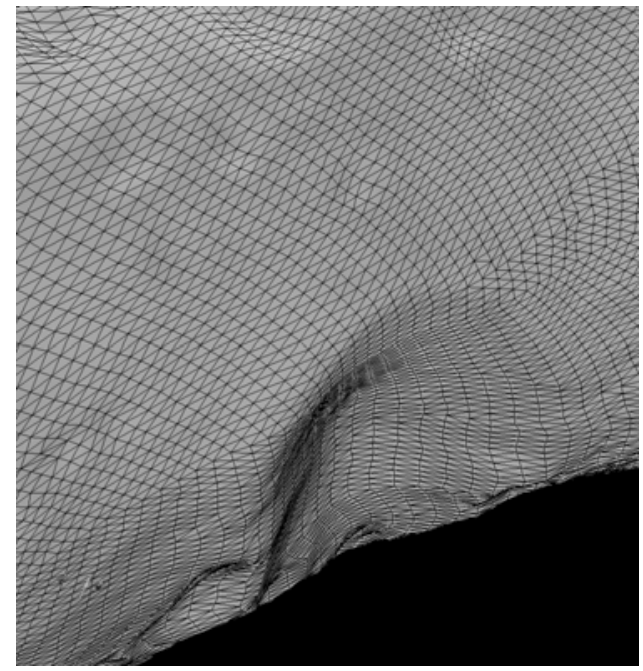

(d) Close-up of triangle patches on ERos's surface, average triangle area $22982 \mathrm{~m}^{2}$, average edge length approx. 230 metres [5].

Abbildung 4: Surface triangulations of small bodies Eros, ItokAwA and Phoвos. Average edge lengths are calculated from the average triangle area, assuming equilateral triangles. Thus, the values for average edge lengths are of correct order of magnitude. 


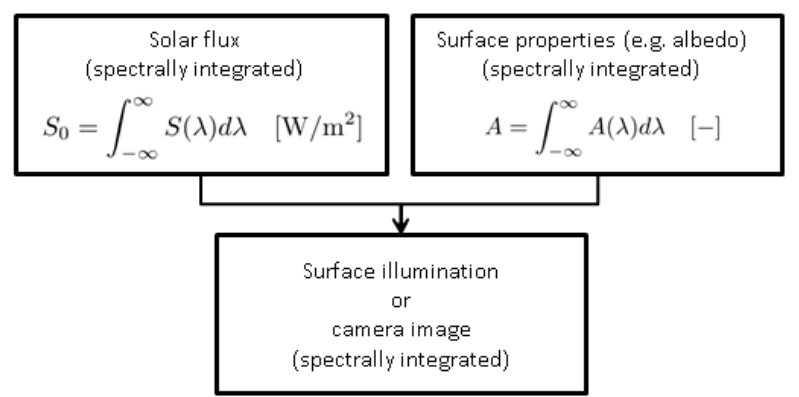

Abbildung 5: Generation of spectrally integrated images. All relevent parameters such as solar flux or optical properties of the surface are averaged and then used in the algorithm.

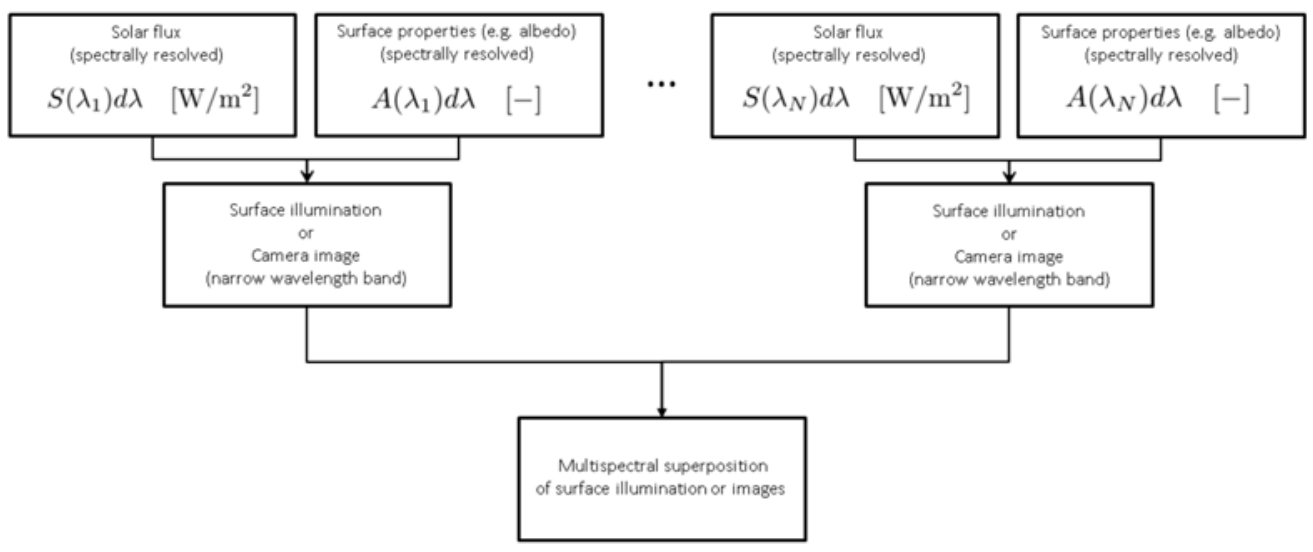

Abbildung 6: Multispectral superposition of surface illumination or camera images. Surface illumination levels or camera images are calculated for $N$ wavelengths resp. wavelength bands (or arbitrary width) and then added. This approach is numerically more expensive but yields more accurate results. 


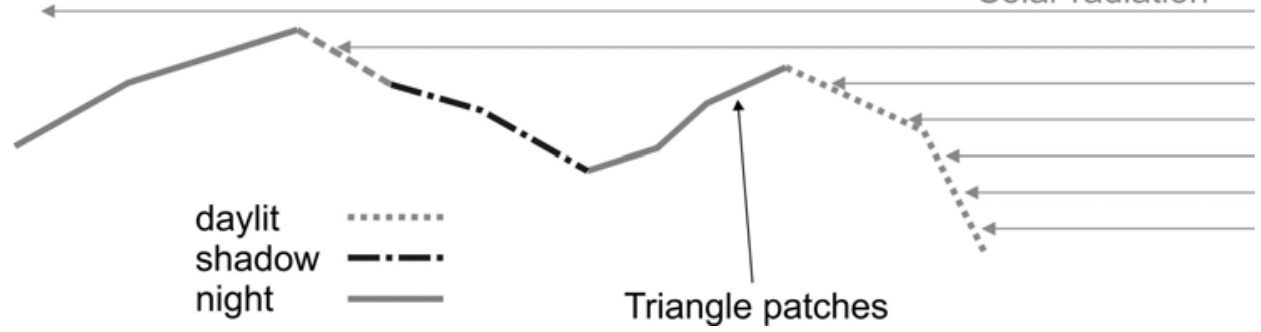

Abbildung 7: Direct radiation on the body surface. There are three types of triangles: day, night and shadow.

\subsection{Direct radiation}

Direct solar radiation is calculated using raytracing on a body-fixed cartesian grid. At the beginning of all calculations, a cartesian grid with a user-defined number of boxes in x-, y-, $\mathrm{z}$-direction is generated. the boundaries of this grid coincide with the axis-aligned bounding box $(\mathrm{AABB})$ of the body under consideration. The home box of a triangle is referred to as the box that the center of gravity of this triangle is located in. To determine which triangle patches are daylit or in shadow (see fig. 7), the solar ray is traced through the grid (see fig. 7). The ray is not tested against intersection with all surface patches but only with those in the respective grid cell (starting in the triangle's home box). If there is no intersection in an individual grid cell, the ray proceeds to the next and the triangle patches in that cell are tested.

During the algorithm, the following pseudocode is executed:

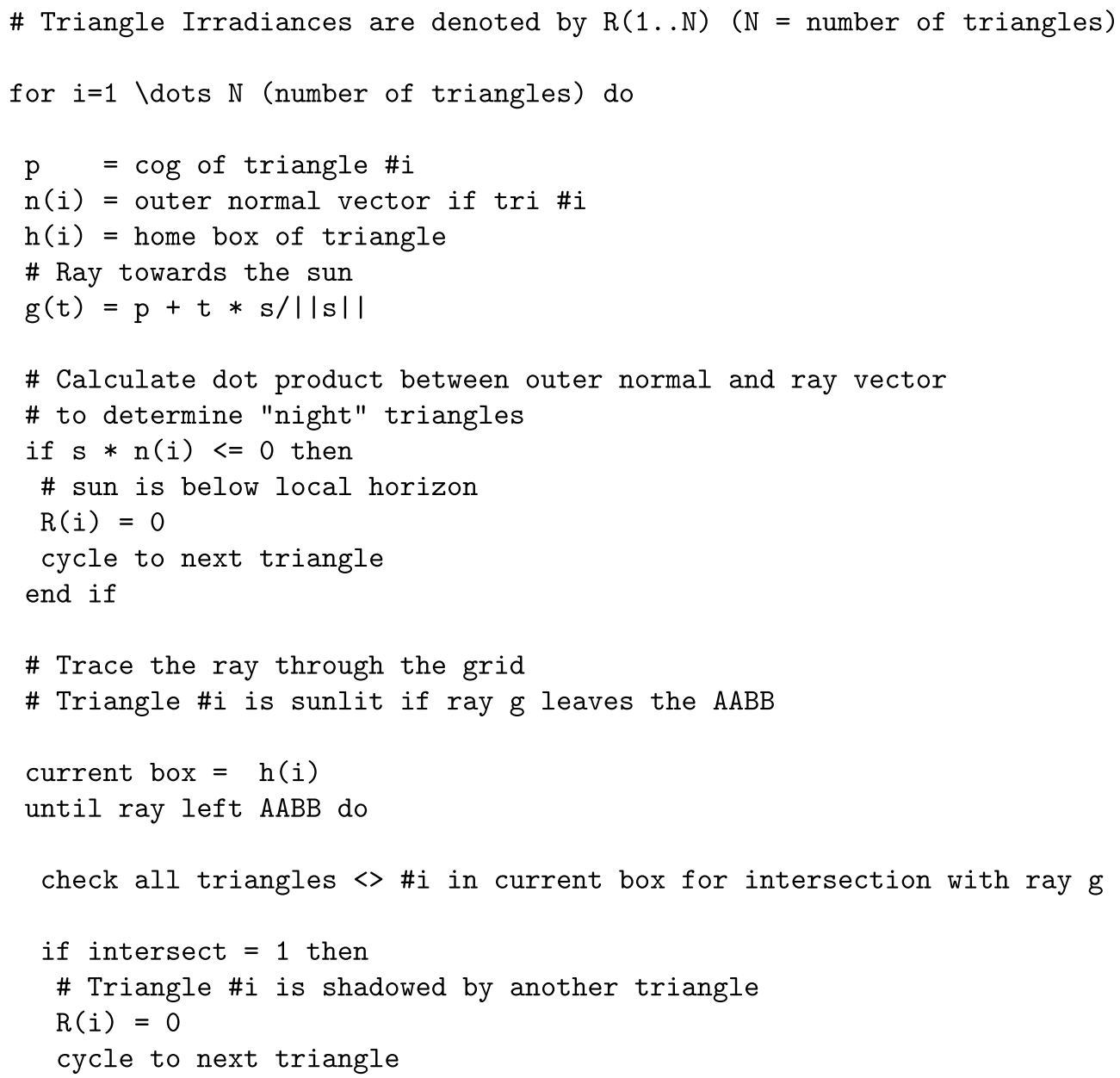




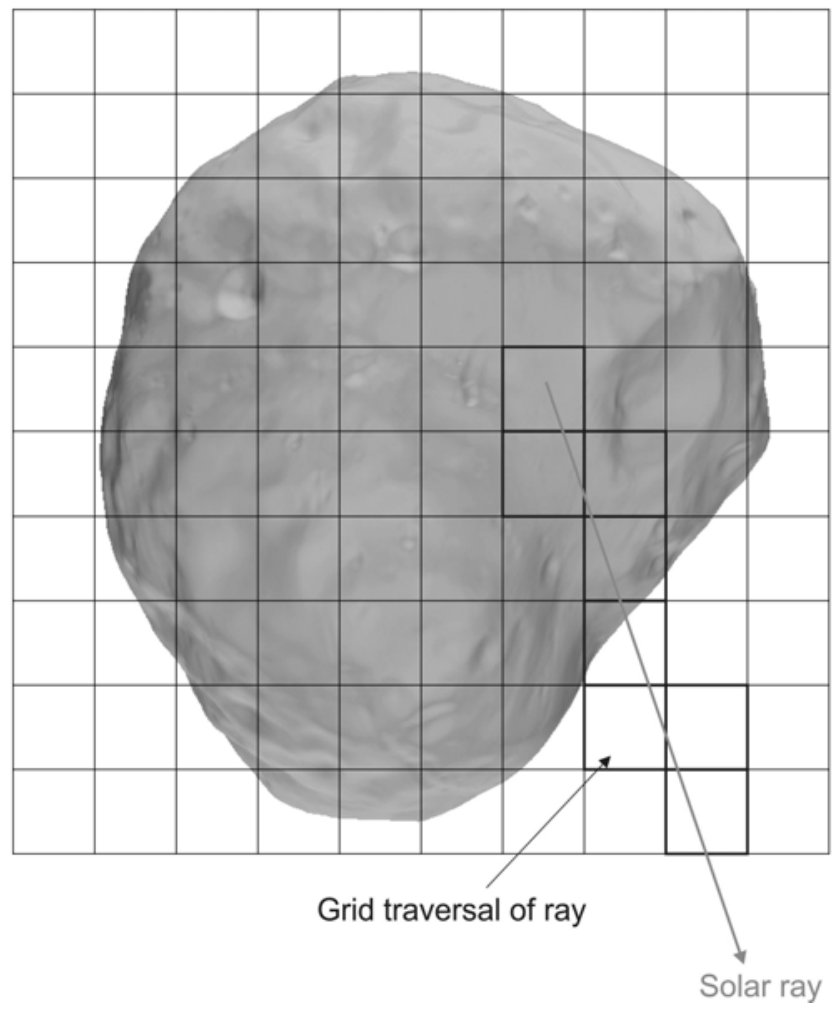

Abbildung 8: Concept of ray-grid traversal. The ray is not tested against intersection with all surface patches but only with those in the respective grid cell. If there is no intersection in an individual grid cell, the ray proceeds to the next and the triangle patches in that cell are tested. 


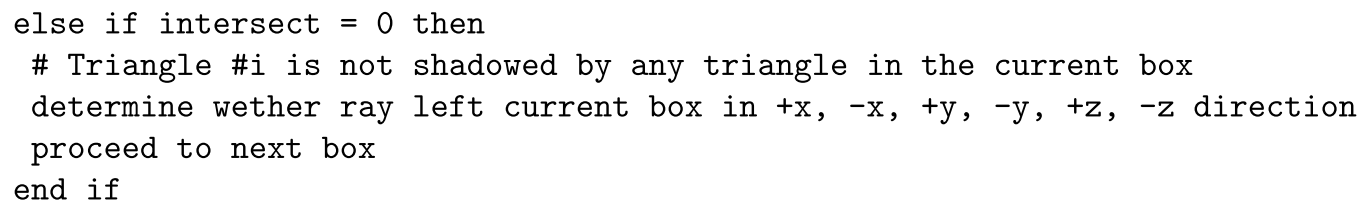

At the end of the algorithm, the following irradiance values $R(i), i=1, \ldots, N$ have been calculated from the solar flux vector $\vec{s}$ and the triangles' outer normal vectors $\vec{n}(i)$ :

$$
\begin{aligned}
\mu & =\langle\vec{s}, \vec{n}(i)\rangle \\
R(i) & =\left\{\begin{array}{lll}
\mu & , \quad \mu \geq 0, \text { i.e. tri is daylit, sun above local horizon } \\
0 & , & \mu<0, \text { i.e. sun below local horizon } \\
0 & , & \text { triangle is shadowed by another triangle }
\end{array}\right.
\end{aligned}
$$

\subsection{Backscatter and Diffuse radiation}

Backscattered light (onto the camera) as well as diffuse radiation (illuminating areas on the body surface that are shadowed in direct light) can be calculated as totally diffuse, i.e. Lambertian reflectance. Alternatively, scattering according to the Hapke reflectance model can be applied ([8]).

The Hapke bidirectional reflectance model has been widely used for modeling of atmosphereless planetary soil surfaces covered with regolith. This covers e..g the lunar surface and the surface of Mercury as well as surfaces of small bodies liek Phobos, Vesta, Ceres etc. Most of those surfaces are characterized by low single-scattering albedos and low degrees of anisotropies. However, effects such as opposition surge might occur, that is a strong increase in backscattered irradiance for ophase angles of approximately zero. This effect can be accounted for in the Hapke model by a "hot spot" correction, parameterized by (angular) width and peak height. Hapke build his model on approximate H-functions developed by Chandrasekhar [8] and derives from this a simple paerameterization of the radiation that is multiply scattered in the sub-surface soil region. From this, a bidreictional reflectance function is dreived that describes apparent reflectance of the soil surface.

Diffuse scattering can be accounted for up to arbitrary orders of scattering by using the method of radiosity ([6], [1], [19], [10]). Radiosity is based on the concept of conservation of energy, i.e. indicent energy flux on a triangle equals outgoing flux. Radiosity quantitifies this idea.

The scattered radiative power $d E$ into solid angle $d \omega$ from a surface patch $d A$ as seen from angle $\phi$ against the surface normal is given by:

$$
d E=I \cos (\phi) d \omega
$$

Here, $I$ is the constant intensity of radiation in all directions. Given diffuse scattering, the radiated power $P$ (in $\mathrm{W} / \mathrm{m}^{2}$ ) of a surface patch $d A$ (here: a triangle patch), consists of two contributions, the self-emitted power $E$ as well as the reflected power $\rho R([6])$ : 


$$
P_{i}=E_{i}+\rho_{i} R_{i} \quad, \quad i=1, \ldots, N
$$

Here, $\rho \in[0,1]$ is the reflectivity (albedo) of the surface, being 0 for a totally black surface and 1 for perfact diffuce reflection. The incident flux $R_{i}$ is the sum of all radiated powers of all other triangles weighted by form factors $F_{i j}$ that quantifiy the mutual visibility and viewing geometry:

$$
\begin{aligned}
R_{i} & =\sum_{j=1, j \neq i}^{N} P_{i} F_{i j}, \quad 1, \ldots, N \\
P_{i} & =E_{i}+\rho_{i} \sum_{j=1, j \neq i}^{N} P_{i} F_{i j} \quad, \quad 1, \ldots, N
\end{aligned}
$$

Given triangles \#i and \# $\mathrm{j}$, let $\theta_{i}$ and $\theta_{j}$ be the angles between the respective outer normal vectors $\vec{n}_{i}, \vec{n}_{j}$ and the line connecting the centers of gravity of the two triangles. Let $r$ be the distance of the cog's. We consider the triangles small w.r.t. to the surface of the small body (typically, the surface is patched with a couple of thousand triangles) as well as plane (this is trivial). Thus, integration over the triangle surfaces is not necessary and the following simplified form of the form factor calculation can be used:

$$
F_{i j}=\left\{\begin{array}{cl}
\frac{\cos \left(\theta_{i}\right) \cos \left(\theta_{j}\right)}{\pi r^{2}} & , \quad \text { tri } \# \mathrm{j} \text { is visible from tri } \# \mathrm{i} \\
0 & , \quad \text { tri } \# \mathrm{j} \text { is not visible from tri } \# \mathrm{i}
\end{array}\right.
$$

Note that

$$
A_{i} F_{i j}=A_{j} F_{j i}
$$

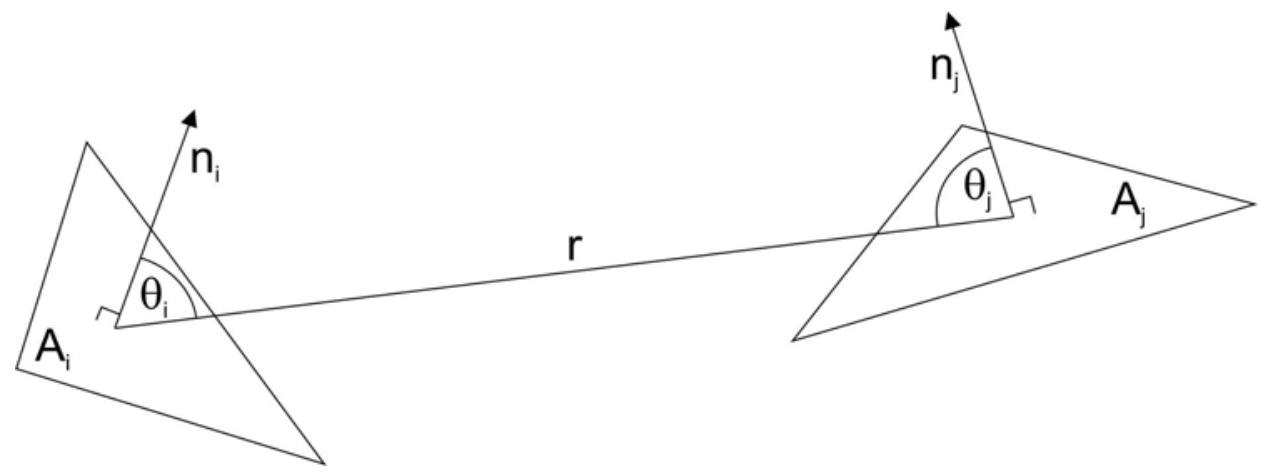

Abbildung 9: Calculation of form factors $F_{i j}$.

Equation (6) constitutes a system of linear equations in $N$ unknowns, the total outbound radiative powers $P_{i}$ of the individual triangle patches. Since $N$ can be large, it is numerically unfavourable to handle the $N^{2}$ coefficient matrix and solve the system directly. It is sparse, so that iterative solvers are favourable. One of those is the JACOBI method. It is easily parallelizable and has the convenient feature that the iterations correspond to the indivual orders of scattering, i.e. after the $k$-th iteration, scattering of order up to $k$ is accounted for. Usually, iterations are ended after a predefined number $K_{0}$ or after convergence, i.e. change in the $P_{i}$ 's below a certain threshold. 


$$
\begin{aligned}
& k=1 \ldots K_{0} \\
& \quad i=1 \ldots N \\
& \quad S_{i}=\left(E_{i}+\rho_{i} \sum_{j=1, j \neq i}^{N} P_{i} F_{i j}\right) /\left(1-\rho_{i} F_{i j}\right) \\
& i=1 \ldots N \\
& \quad P_{i}=S_{i}
\end{aligned}
$$

Note that for the similar Gauss-Seidel method, step (10) is to be omitted and $S_{i}$ to be replaced by $B_{i}$ in step (9).

The Hapke reflectance model follows basically the same algorithm except that the isotropic intensity $I$ in (3) needs to be replaced by the scattering phase function $P$ of the form:

$$
\begin{aligned}
P\left(\mu, \mu_{0}, \Omega\right) & =\frac{\omega}{4\left(\mu_{0}+\mu\right)}\left[P(\Omega)(1+B(\Omega))+H(\mu) H\left(\mu_{0}\right)-1\right] \\
H(x) & =\frac{1+2 x}{1+2 x \sqrt{1-\omega}} \\
B(x) & =\frac{B_{0}}{1+\tan (x / 2) / h}
\end{aligned}
$$

Here, $\mu, \mu_{0}, \Omega$ denote the cosine of the inbound ray and the outbound ray and the phase angle between inbound and outbound ray, respectively. Additional surface parameters are reprsented by $\omega, B_{0}, h$, the single scattering albedo and the height and angular width of the hot-spot correction.

\section{Remark on Multiple Bodies}

The algorithm does not distinguish between triangle patches of one or the other body. An arbitrary number of bodies can be handled simultaneously, i.e. illumination of one body by one (or more) other(s) can be handled by design. at the beginning of the algorithm, triangle meshes of the individual bodies are read and concatenated into a single triangle mesh. For this mesh, generation of the AABB (see sec. 6.1), direct illumination (sec. 6.1) as well as diffuse illumination up to arbitrary orders of scattering (sec. 6.2) is carried out. The same applies for mutual shadowing, this is in fact a part of the calculation of direct illumination. 
Mesh 1 (data type: tri mesh)

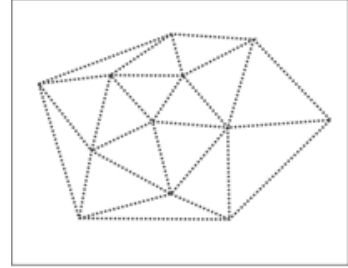

Mesh 2 (data type: tri mesh)
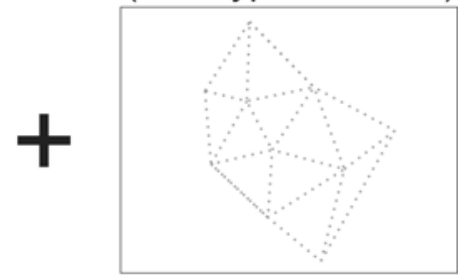

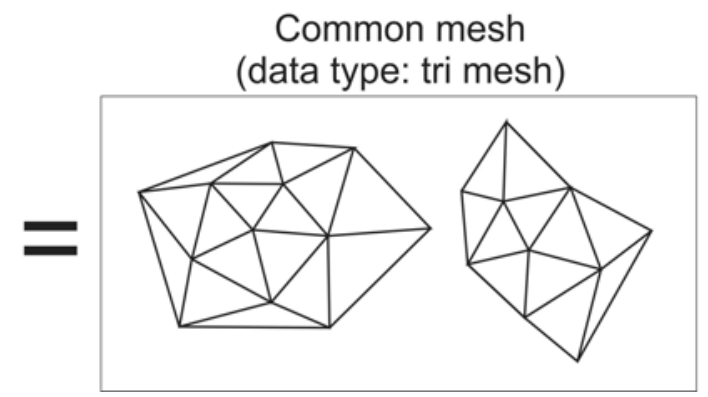

Abbildung 10: Multiple bodies' triangle meshes are internally handled like a single mesh, thus allowing for easy implementation of mutual illumination or shadowing as well selfillumination (diffuse) e.g. in crater walls.

\section{Examplary Applications}

\subsection{Example: Phobos}

The Martian moon Рновоs has been illuminated, showing shadowing e.g. of crater floors, see fig. 11 and 12. The spatial resolution of this mesh is 49152 triangles, i.e. approximately $278 \mathrm{~m}$ average triangle edge length.

\subsection{Mutual Shadowing}

Figure 13 shows mutual shadowing of two spheres. The two spheres are assumed to have equal radii of approx. $1200 \mathrm{~km}$ (comparable to Pluto, see below) and are equally meshed with 2048 and 4096 nodes and triangles each, respectively. One sphere is located at $(0,0,0)$, whereas the other is located at $(-3000,1000,500)$, solar radiation is assumed to impinge from direction $(1,0,0)$. The shadow of one sphere on the surface of the other is clearly visible, showing discretization artifacts at the border, though. Note that in general, small bodies or lunar surfaces are available in much higher resolution, see fig. 4.

The Pluto-Charon binary system has been considered as second example. Idealized positions of Pluto and Charon have been assumed, i.e. both bodies on $\mathrm{x}$ axis, separated by the real average distance of $19596 \mathrm{~km}$. Radii are $1187 \mathrm{~km}$ and $606 \mathrm{~km}$ for Pluto and Charon, respectively. Figure (10) shows the shadow casted by Charon on Pluto's surface, assuming the sun in direction $(1,0,0)$.

\subsection{Illumination Statistic}

The SLIM illumination model can be used to carry out statistical analysis, yielding the cumulative distribution function (cdf) for the irradiance at differen triangle patches on the body surface. The cumulative distribution function assesses the times the irradiance is above some certain level. Usually, time intervals are calculated w.r.t to some total time, e.g. a full spin period. 


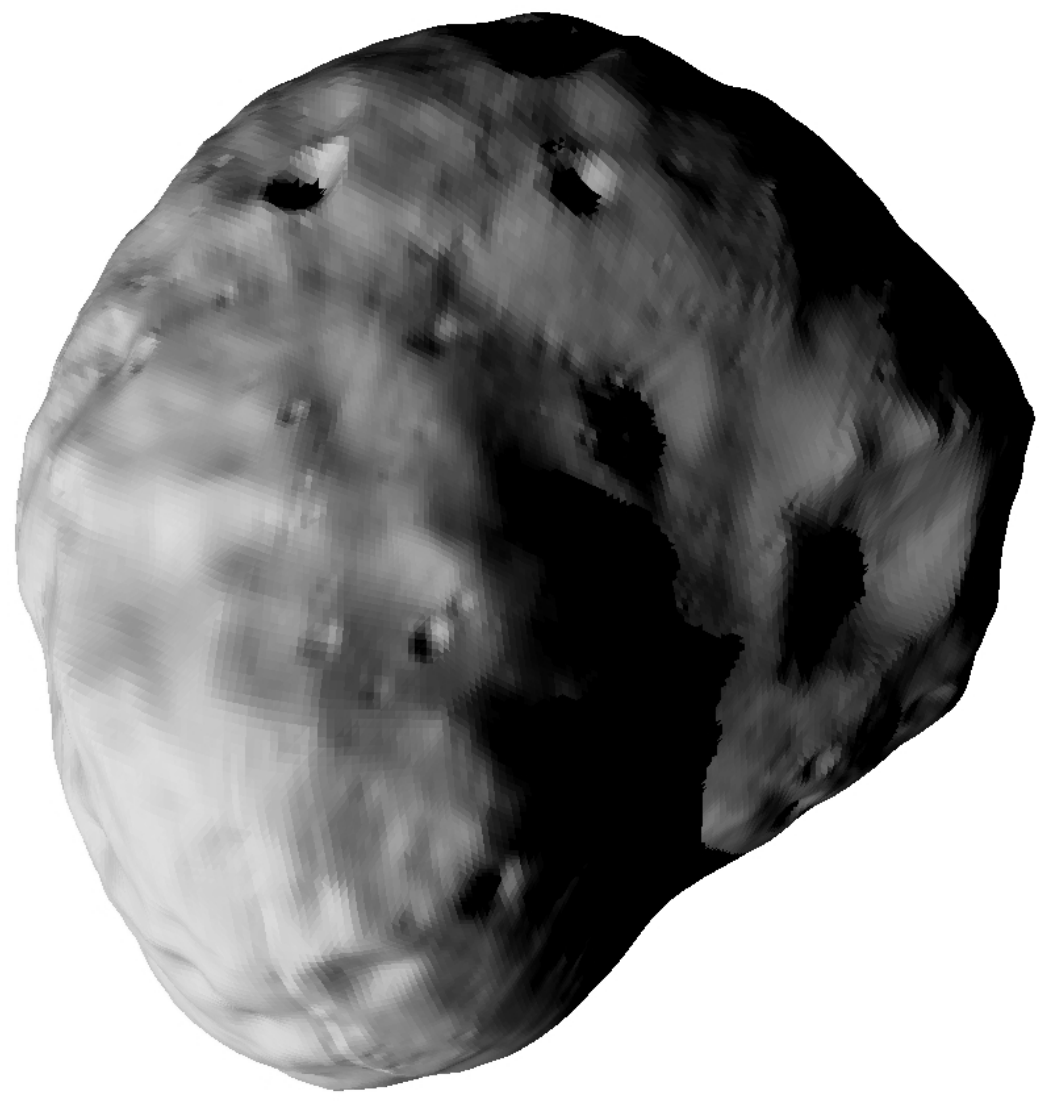

Abbildung 11: Solar illumination of Martian moon Рновоs [5], showing realistic shadowing e.g. of craters.

$$
F_{k}(x) \cdot T_{\text {tot }}=\left\{t \in \left[0, \infty\left[: I_{k}(t) \leq x\right\}\right.\right.
$$

In other words, the irradiance on triangle patch $\# k$ is smaller than some level $x$ for " $F_{k}(x) \%$ " of the total time. Figure 15 shows the results for Pновоs, calculated for a full spin period of $7.65 \mathrm{~h}$ at $1.52 \mathrm{AU}$ heliocentric distance and for zero obliquity. Shown are exemplary cdfs for five different triangles. Note that the maximal irradiance does not exceed approx. 500 $\mathrm{W} / \mathrm{m}^{2}$. 


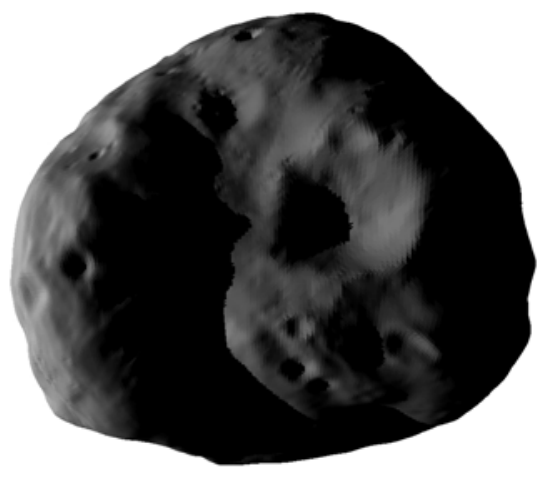

(a) Phoвos [5], solar radiation from left hand side.

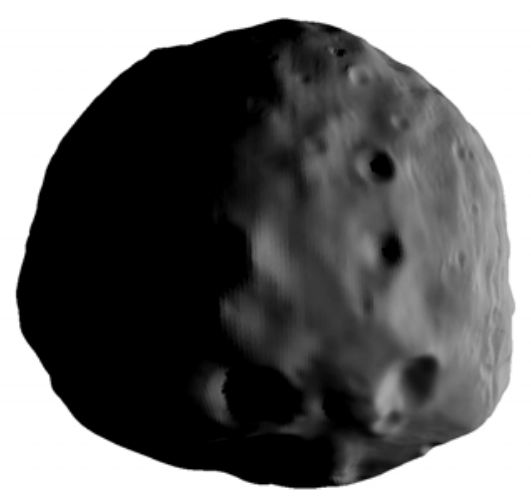

(c) Phовоs [5], solar radiation from right hand side.

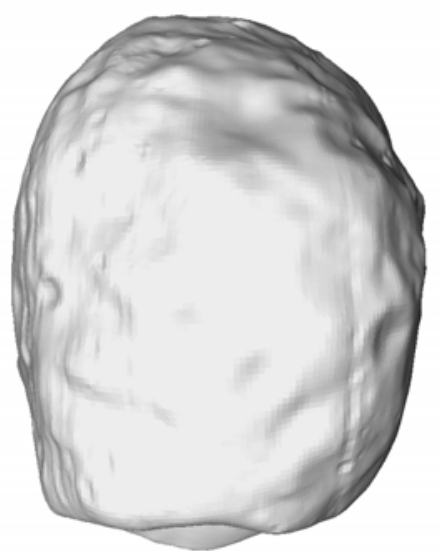

(b) Phoвos [5], solar radiation from front (directed into image plane).

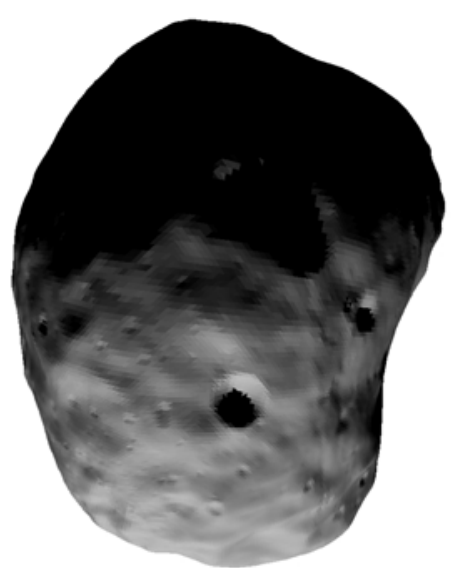

(d) Phoвоs [5], solar radiation from bottom.

Abbildung 12: Solar illumination of Martian moon PHOBOs, showing realistic shadowing in different angles of view. 


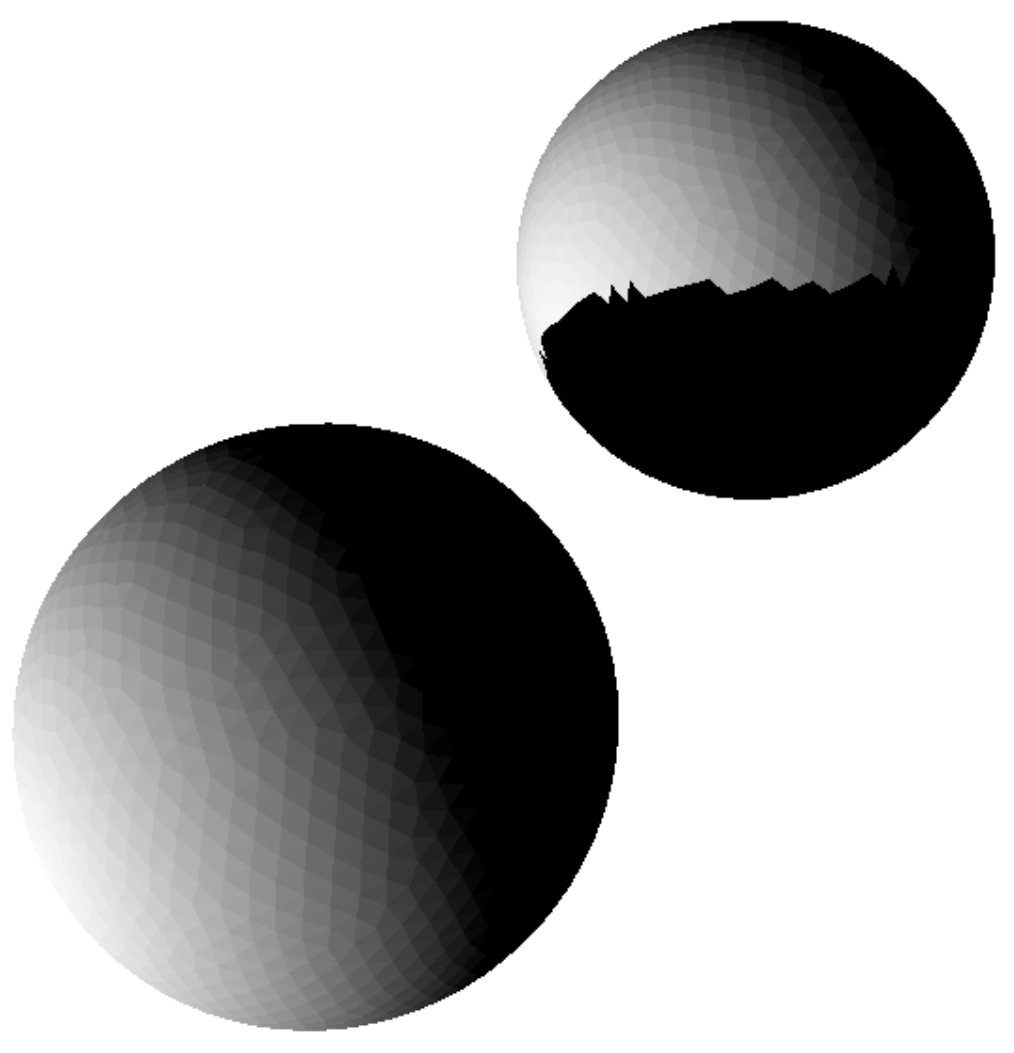

Abbildung 13: Shadowing of two spheres of radius $1200 \mathrm{~km}$, each. Spheres are located at $(0,0,0)$ and $(-3000,1000,500)$, respectively. Direction to sun is $(1 m, 0,0)$.
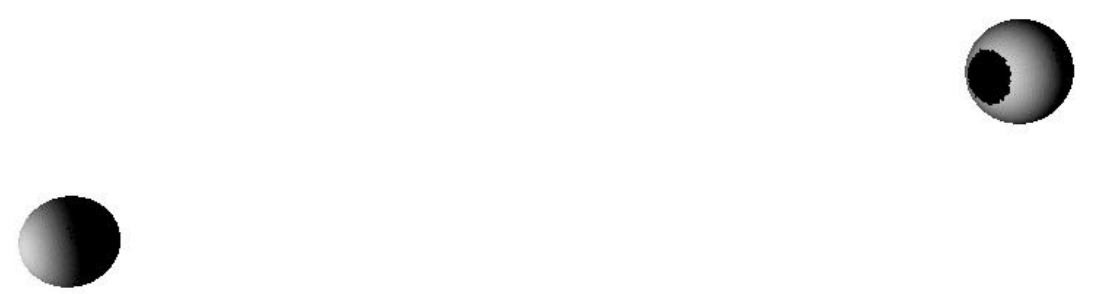

Abbildung 14: Shadowing of Pluto (right) by its largest moon Charon (left) during lunar eclipse (idealized). Both bodies are located on the $\mathrm{x}$ axis, coordinates: Pluto $(0,0,0)$, Charon $(19596,0,0)$, sun vector $(1,0,0)$.

\section{Validation}

A number of publications reported on optical properties of the surface of (4) Vesta as measured using the DAWN framing camera [18], see e.g. [12], [9], [17]. A,so, landmarks estimates from optical (as well as tracking) data have been investigated to derive dynamical properties such as gravity field, spin pole and rotation period [11]. 


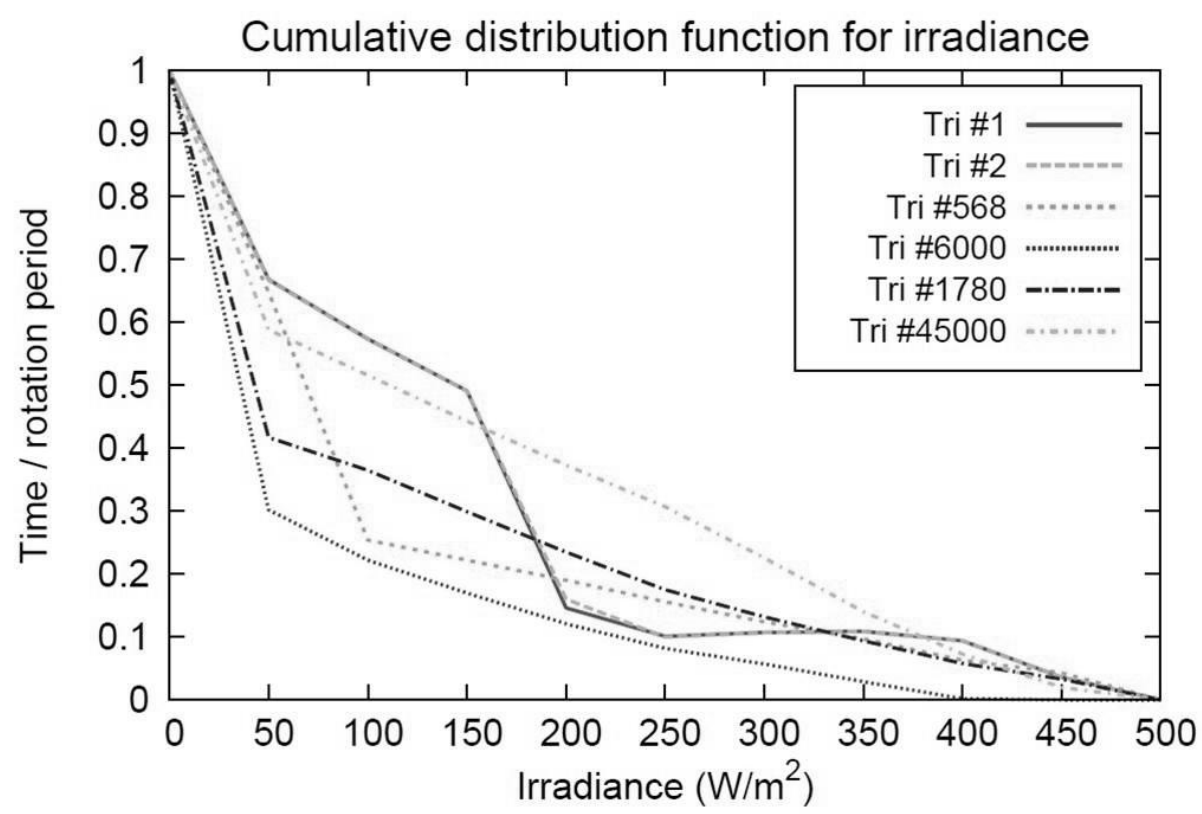

Abbildung 15: Cumulative distribution function for the irradiance at different points (triangle patches) on Рновоs' surface, relative to $T_{\text {tot }}$ being Рновоs' spin period, i.e. $7.65 \mathrm{~h}$. Shown is $F_{k}(x)$, see eq. (14) for five different triangles

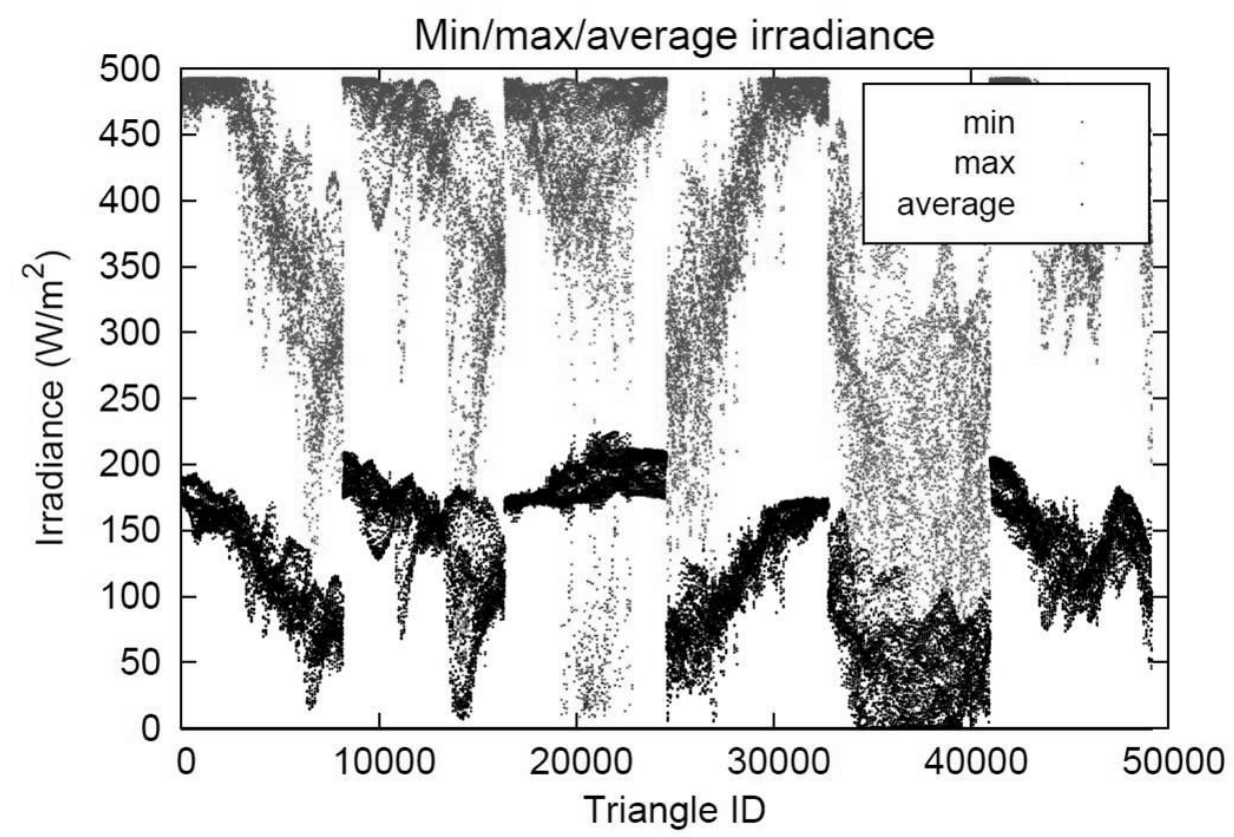

Abbildung 16: Mininmal/maximal/average values for the irradiance at different points (triangle patches) on Рновоs' surface, relative to $T_{\text {tot }}$ being Phoвоs' spin period, i.e. $7.65 \mathrm{~h}$. Note that a number of triangles near the north pole (ID aporox. 20000) is in permanent daylight. Assumed obliquity was zero.

For validation purposes, a number of images acquired by the DAWN Framing Camera FC2 (see figure 18 for basic parameters) has been utilized. Corresponding ephemerides and pointing parameters have been provided by the SPICE toolkit in version N00066, released April 2017 [13].

The validation data set (2665 pictures) is covering the time span of August $11-28$ of the 
DAWN/Vesta survey data set, i.e. the mission phase covering overview pictures of 4 Vesta following the approach phase and followed by the high-altitude mapping orbit (HAMO) and low-altitude mapping orbit (LAMO) phase:

\begin{tabular}{|l|l|}
\hline Time & Mission Phase \\
\hline Jul 16, 2011 & Vesta arrival \\
Aug $11-31,2011$ & Vesta survey phase \\
Sep 29, 2011- Nov 2, 2011 & Vesta first high altitude orbit (HAMO) \\
Dec 12, 2011- May 1, 2012 & Vesta low altitude orbit (LAMO) \\
Jun 15,2012- Jul 25, 2012 & Vesta second high altitude orbit (HAMO) \\
Sep 5,2012 & Vesta departure \\
\hline
\end{tabular}

Tabelle 1: DAWN mission phases in Vesta's vicinity.

During the survey phase, the average distance between the S/C and 4 Vesta is approx. 2720 $\mathrm{km}$, phase angles (angle between line-of-sight DAWN-Vesta and line connecting Vesta and the sun) cover the range from approx. $11 \mathrm{deg}$ to approx. $81 \mathrm{deg}$.

The image resolution is constant at 1024 x 1024 pixels. The Vesta shape model (compare section 4) is available in four different resolutions:

\begin{tabular}{|l|l|l|}
\hline Resolution & No. of triangles & Average edge length \\
\hline coarse & 49152 & $6744.7 \mathrm{~m}$ \\
medium & 196608 & $419.2 \mathrm{~m}$ \\
fine & 786432 & $28.3 \mathrm{~m}$ \\
super-fine & 3145728 & $1.7 \mathrm{~m}$ \\
\hline
\end{tabular}

Tabelle 2: Available resolutions of surface grid of 4 Vesta.

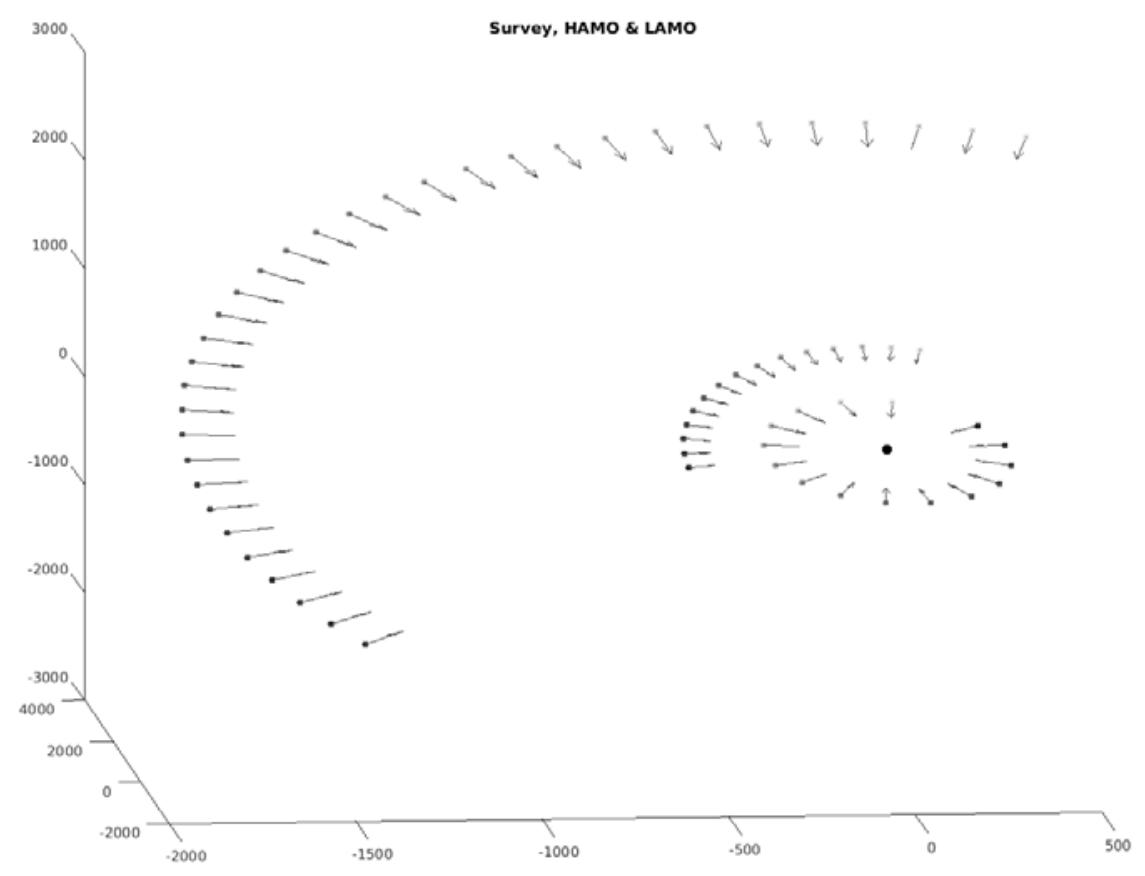

Abbildung 17: Some DAWN S/C positions relative to 4 Vesta during the survey, HAMO and LAMO phase. Orientetions of the camera (FC 2) are shown as red arrows, color of each dot represent time during each mission phase from yellow (early) to blue (later). 


\begin{tabular}{lr}
\hline Parameter & FC \\
\hline Focal Length (mm) & 150.0 \\
Aperture (mm) & 19.9 \\
f/ratio & $\mathrm{f} / 8$ \\
IFOV (microrad/pixel) & 93.3 \\
Field of view (degree) & \\
$\quad$ Cross-track & 5.46 \\
$\quad$ Along-track & 5.46 \\
\hline
\end{tabular}

Abbildung 18: Basic parameters of Framing Camera 2 of the DAWN mission [18]. For the study presented here, data of the camera \#ID 2 have been utilized.

\begin{tabular}{llllll}
\hline $\begin{array}{l}\text { Channel } \\
\text { No. }\end{array}$ & $\begin{array}{l}\text { Center } \\
\text { wavelength }[\mathrm{nm}]\end{array}$ & $\begin{array}{l}\text { Bandwidth } \\
{[\mathrm{nm}]}\end{array}$ & $\begin{array}{l}\text { Trans- } \\
\text { mission } \\
{[\%]}\end{array}$ & $\begin{array}{l}\text { Filter-wheel } \\
\text { position }\end{array}$ & $\begin{array}{l}\text { Thickness } \\
{[\mathrm{mm}]}\end{array}$ \\
\hline 1 & polychromatic & $\begin{array}{l}450 \pm 10 \text { to } \\
920 \pm 10\end{array}$ & 98 & F1 & $6.00 \pm 0.05$ \\
2 & $430 \pm 2$ & $40 \pm 5$ & $>75$ & F8 & $2.00 \pm 0.05$ \\
3 & $550 \pm 2$ & $40 \pm 5$ & $>75$ & F2 & $5.90 \pm 0.05$ \\
4 & $650 \pm 2$ & $40 \pm 5$ & $>75$ & F7 & $6.60 \pm 0.05$ \\
5 & $750 \pm 2$ & $40 \pm 5$ & $>75$ & F3 & $6.40 \pm 0.05$ \\
6 & $830 \pm 2$ & $40 \pm 5$ & $>75$ & F6 & $5.90 \pm 0.05$ \\
7 & $920 \pm 2$ & $40 \pm 5$ & $>75$ & F4 & $5.30 \pm 0.05$ \\
8 & $980 \pm 2$ & $80 \pm 5$ & $>75$ & F5 & $4.80 \pm 0.05$ \\
\hline
\end{tabular}

Abbildung 19: Filter settings of Framing Camera 2 of the DAWN mission [18]. For the study presented here, measurements using the clear filter have been utilized.
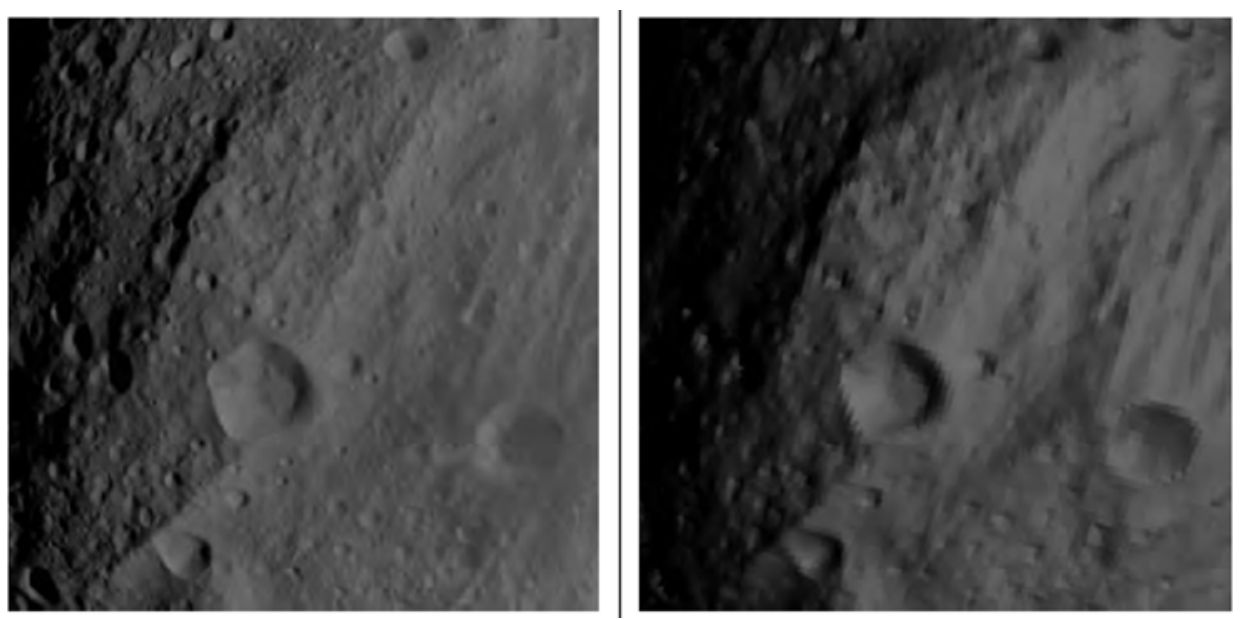

Abbildung 20: Left: Simulated image, right: real image as acquired by DAWN Framing Camera FC2. Image ID ..

\subsection{Statistical Analysis}

Simulated and acquired images are compared by utilizing the irradiance probability density distribution (IPDF). Measured respectively simulated irradiances have been binned into 10 $\mathrm{W} / \mathrm{m}^{2}$ bins ranging from 0 to approx. $290 \mathrm{~W} / \mathrm{m}^{2}$. Note that the solar constant at 4 Vesta (approx. 2.27 AU from the sun during the DAWN survey phase) is approximately $267 \mathrm{~W} / \mathrm{m}^{2}$. An examplary IPDF is shown in figure 22.

The validation approach followed in this study is based on the comparison of simulated and 

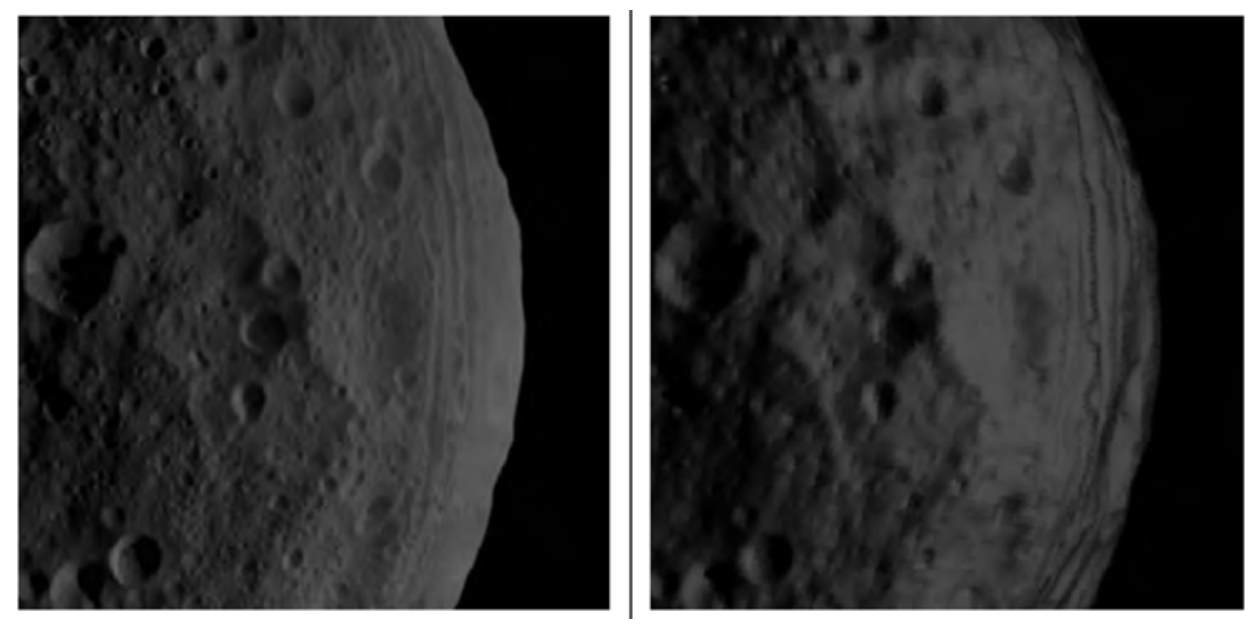

Abbildung 21: Left: Simulated image, right: real image as acquired by DAWN Framing Camera FC2. Image ID FC21B0005907-11232212051F1B.FIT.

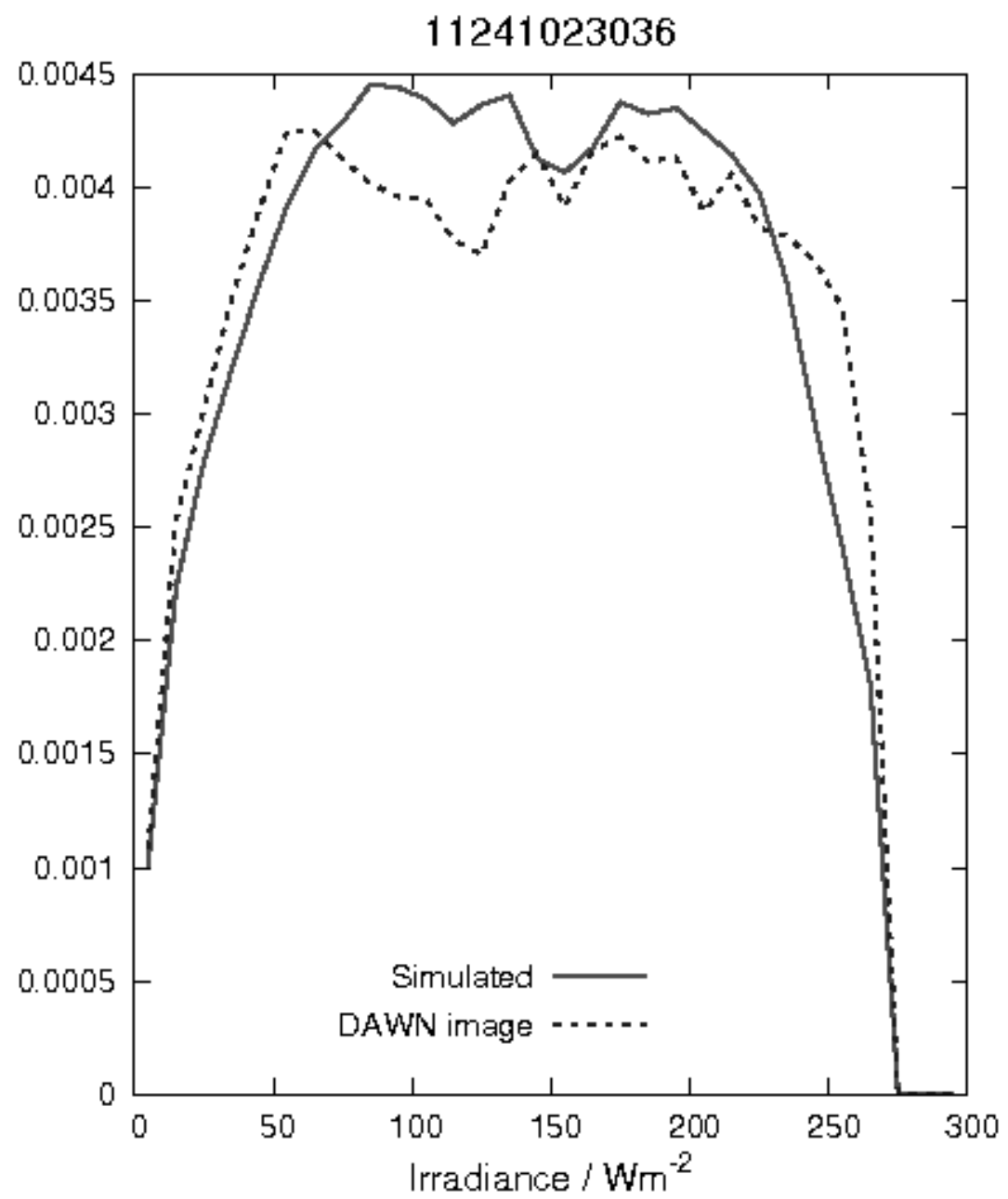

Abbildung 22: Exemplary irradiance probability density function of image ID 11241023036. DAWN image acquired on August 31. The IPDF shows an average irradiance of approx. 151 $\mathrm{W} / \mathrm{m}^{2}$ and minimal and maximal values of 0 . (black pixel or deep shadow) and $260 \mathrm{~W} / \mathrm{m}^{2}$, respectively. Note that the irradiance bins are $10 \mathrm{~W} / \mathrm{m}^{2}$. 
acquired (measured) IPDF. This is done using a $\chi^{2}$ test. Compare [...] for an extensive textbook level introduction. The test method is briefly outlined as follows. Assume a given IPDF with frequencies $f_{1}, \ldots, f_{n}$ for the various irradiance bins. This is represented by the IPDF of the measured image. Assume $p_{1}, \ldots, p_{n}$ be the frequencies of the IPDF to be compared with. Given $N$ samples pixels in this case), the deviation between the two IPDF can be quantified by the $\chi_{s}^{2}$ value:

$$
\chi_{s}^{2}=\sum_{k=1}^{N} \frac{\left(f_{k}-N p_{k}\right)^{2}}{N p_{k}}
$$

The $\chi_{s}^{2}$ test statistic is an overall measure of how close the observed frequencies $f_{k}$ are to the expected frequencies $p_{k}$. Obviously, the 'null hypothesis' (PDF and independent, i.e. simulated and measured image are not similar at all) is rejected if $\chi^{2}$ is large, because this means that observed frequencies and expected frequencies are far apart. A quantification of 'large' is acquired by the $\chi^{2}$ probability density function:

$$
f(x)=\frac{x^{N / 2-1} e^{-x / 2}}{2^{N / 2} \Gamma(N / 2)}
$$

Here, $N$ represents the degrees of freedom, identical with the number of pixels in our case. The $\chi^{2}$ curve is used to judge whether the calculated test statistic is large enough. We reject the null hypothesis if the test statistic is large enough so that the area beyond is less than 0.05 :

$$
P\left(\chi^{2} \geq \chi_{s}^{2}\right)=\int_{\chi_{s}^{2}}^{\infty} f\left(\chi^{2}\right) d\left(\chi^{2}\right)
$$

This test is done for every picture and for every resolution available for the Vesta surface grid (see table 2). As can be seen in figures $23-26$, the majority of the test cases yield values of $P\left(\chi^{2} \geq \chi_{s}^{2}\right)<0.05$, i.e. the null hypothesis is rejected. Note that the null hypothesis is acutally that the underlying IPDF of the acquired and simulated images are different. Thus, the reliability of the algorithm is good.

Additionally, the quality of the simulated images, taking the number of test cases with $P\left(\chi^{2} \geq \chi_{s}^{2}\right)<0.05$ as metric - improves for increasing number of surface triangles. This is reasonable, since the real surface can be considered as the $n_{T}=\infty$ limit case and the effect of surface discretization is decreased for increasing values of $n_{T}$. 


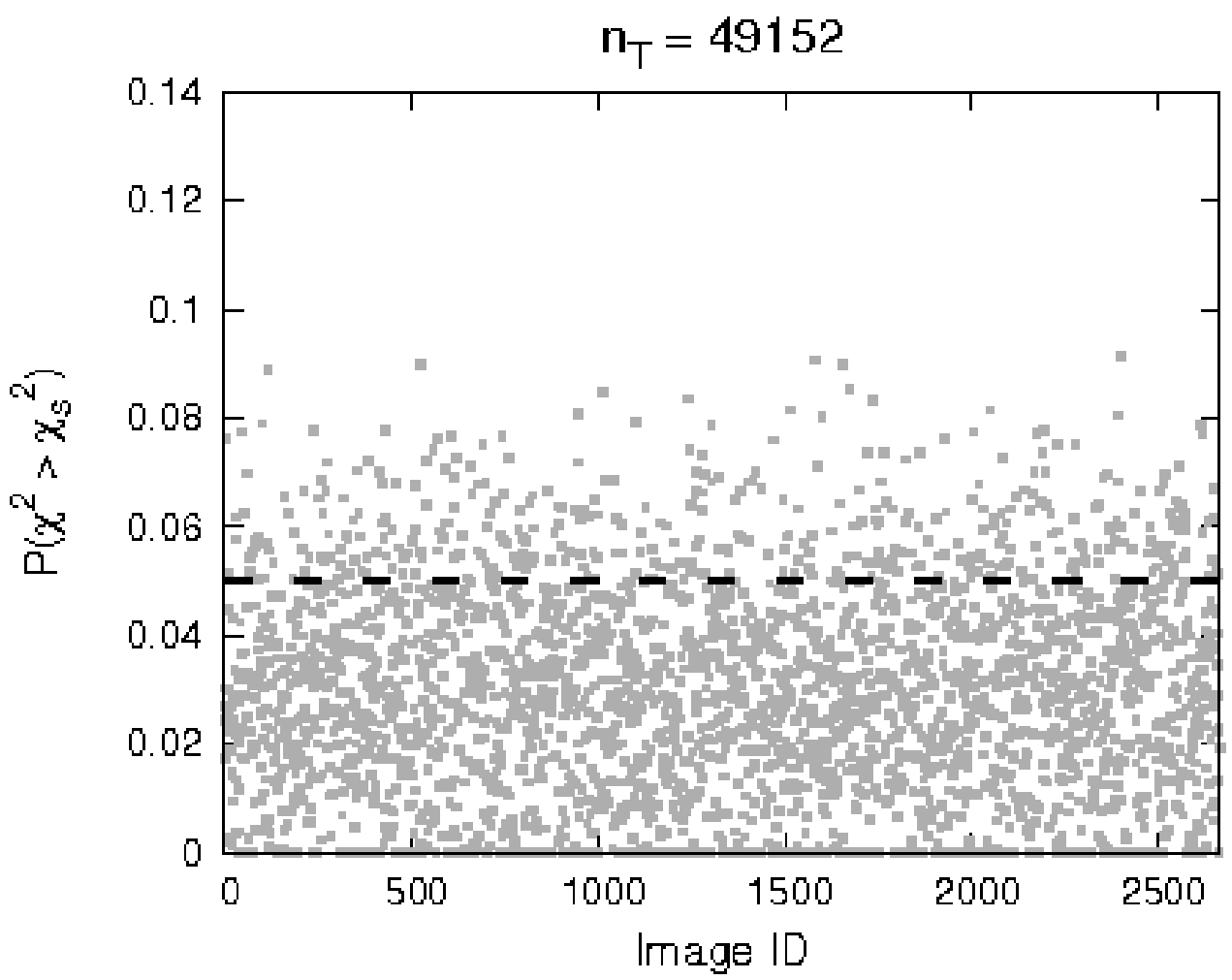

Abbildung 23: Results of $\chi^{2}$ test, 49152 surface triangles.

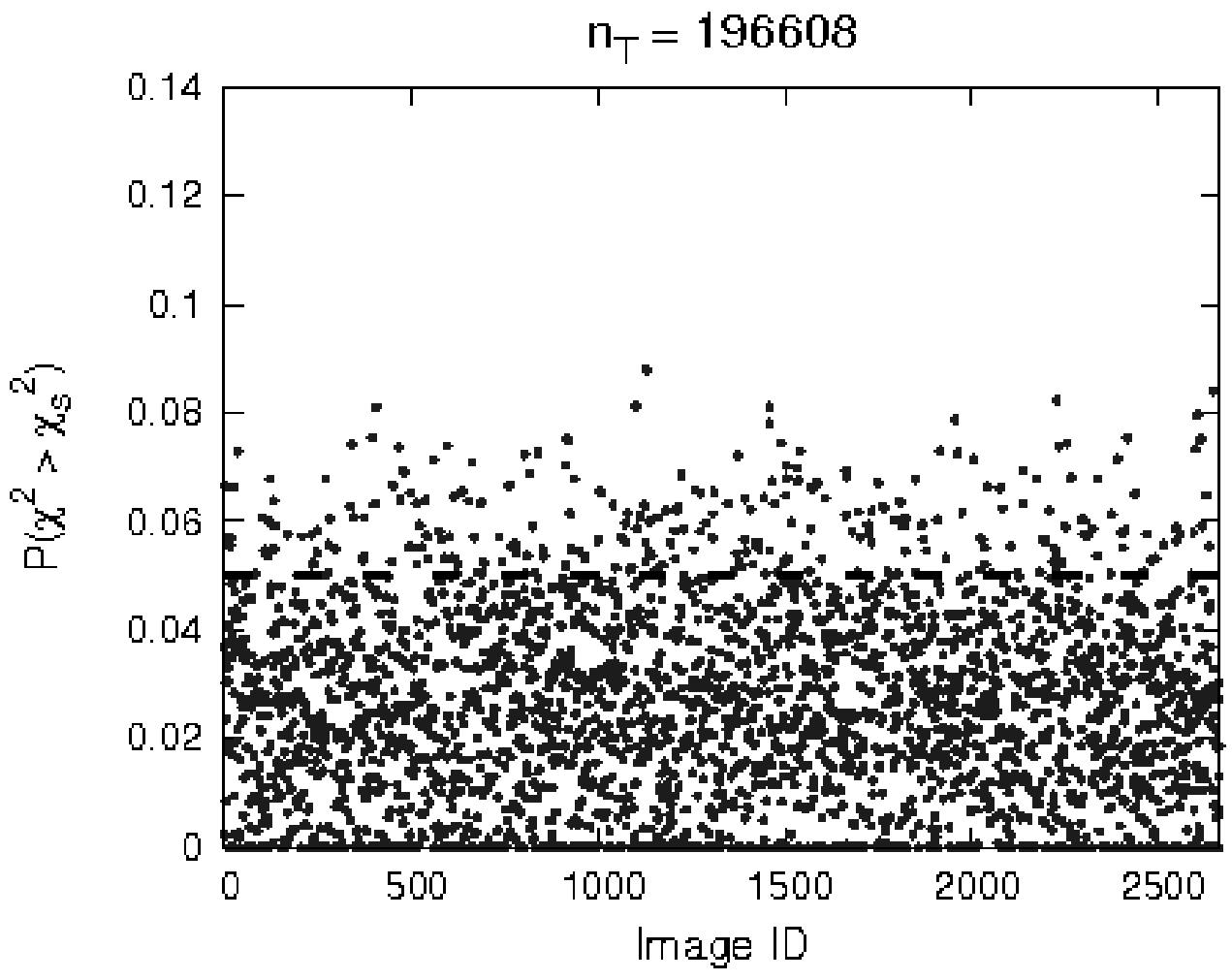

Abbildung 24: Results of $\chi^{2}$ test, 196608 surface triangles. 


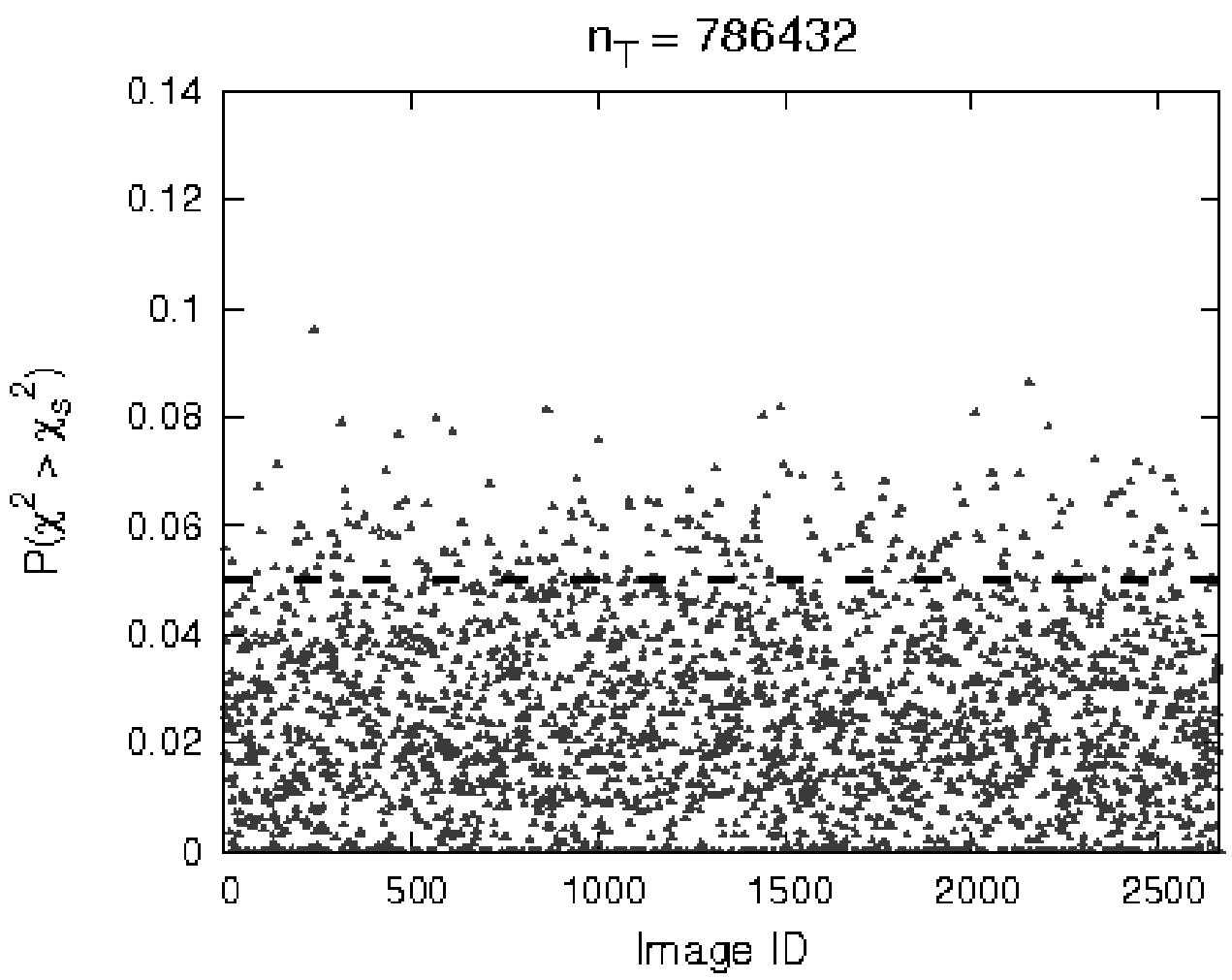

Abbildung 25: Results of $\chi^{2}$ test, 786432 surface triangles.

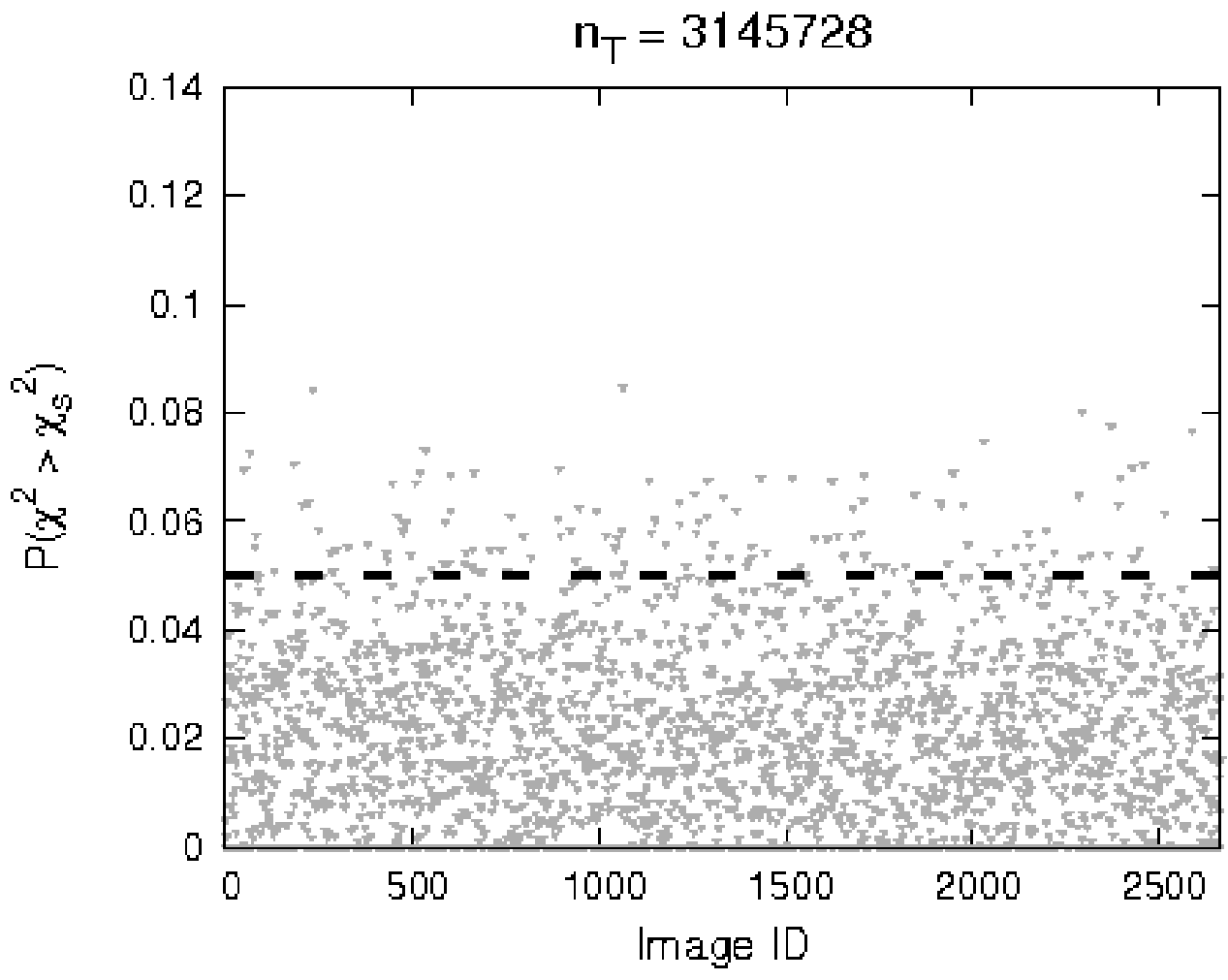

Abbildung 26: Results of $\chi^{2}$ test, 3145728 surface triangles. 


\section{Conclusions and outlook}

An efficient raytracing software for simulation of camera images as well as illumination conditions of small bodies as well as planetary surfaces has been implemented. Direct as well as indirect illumination as well as illumination of multiple bodies are possible. Different bidirectional reflectance functions are implemented. This includes the four-parameter Hapke model.

The illumination model has been validated using a $\chi^{2}$ statistical test to compare simulated images with a large number of DAWN acquired images. Good statistical match is found in general.

Main benefits of the simulation suite are its easy implementation and the option to use it as forward model in an inverse problem solver to derive optical properties of the surface of small bodies or planetary surfaces (without atmosphere).

Using this illumination model, optical parameters can be extracted from the comparison of simulated and observed radiances and applications of an optimization algorithm to those data. Images in different spectral bands can be easily computed by restriction of the solar spectrum to the spectral region under consideration. This way, dependence of the spectral parameters on wavelength can be examined.

This has non-zero intersection with inverse problem theory, as the measured images might be noisy and the simulated images are restricted due to finite discretization of the surface, either a small body of any other planetary surface. 


\section{Literatur}

[1] R. L. Cook and K. E. Torance. A relectance model for computer graphics. ACM Transactions on Graphics, 1(1), 1982.

[2] ESA. Asteroid impact mission: Didymos reference model. Technical Report 2.1, ESA, 2015.

[3] R. Gaskell, J. Saito, M. Ishiguro, T. Kubota, T. Hashimoto, N. Hirata, S. Abe, O. Barnouin-Jha, and D. Scheeres. Gaskell itokawa shape model v1.0. hay-a-amica5-itokawashape-v1.0. NASA Planetary Data System, 2008.

[4] R.W. Gaskell. Gaskell eros shape model v1.0. near-a-msi-5-erosshape-v1.0. NASA Planetary Data System, 2008.

[5] R.W. Gaskell. Gaskell phobos shape model v1.0. vo1-sa-visa/visb-5-phobosshape-v1.0. NASA Planetary Data System, 2011.

[6] C. M. Goral, K. E. Torrance, D. P. Greenberg, and B. Battaile. Modeling the interaction of light between diffuse surfaces. Computer Graphics, 18(3), 1984.

[7] E. Graciano, J. and. Chester. Autonomous rover path planning and reconfiguration. In 11th Symposium on Advanced Space Technologies in Robotics and Automation (ASTRA), European Space Agency, Noordwijk, Netherlands., 2011.

[8] B. W. Hapke. Bidirectional reflectance spectroscopy. J. Geophys. Res., 86:3039-3054, 1981.

[9] M. D. Hicks, B. J. Buratti, K. J. Lawrence, J. Hillier, J.-Y. Li, V. Reddy, S. Schröder, A. Nathues, M. Hoffmann, L. le Corre, R. Duffard, H.-B. Zhao, C. Raymond, C. Russell, T. Roatsch, R. Jaumann, H. Rhoades, D. Mayes, T. Barajas, T.-T. Truong, J. Foster, and A. McAuley. Spectral diversity and photometric behavior of main-belt and nearEarth vestoids and (4) Vesta: A study in preparation for the Dawn encounter. Icarus, 235:60-74, June 2014.

[10] J. T. Kajiya. The rendering equation. In Comm. ACM, SIGGRAPH 1986, volume 5-6, 1986.

[11] A. S. Konopliv, S. W. Asmar, R. S. Park, B. G. Bills, F. Centinello, A. B. Chamberlin, A. Ermakov, R. W. Gaskell, N. Rambaux, C. A. Raymond, C. T. Russell, P. Smith, D. E. Tricarico, and M. T. Zuber. The vesta gravity field, spin pole and rotation period, landmark positions, and ephemeris from the dawn tracking and optical data. Icarus, 240:103-117, 2013.

[12] J.-Y. Li, L. B. Le Corre, S. E. Schröder, V. Reddy, B. W. Denevi, B. J. Buratti, M. Mottola, S. nd Hoffmann, P. Gutierrez-Marques, A. Nathues, C. T. Russell, and C. A.W. Raymond. Global photometric properties of asteroid (4) vesta observed with dawn framing camera. Icarus, 226(2):1252-1274, 2013.

[13] Navigation and Ancillary Information Facility (NAIF). Spice - an observation geometry system for space science missions. Technical report, National Aeronautics and Space Administration (NASA), 2017.

[14] Tompkins P., A. Stentz, and D. Wettergreen. Global path planning for mars rover exploration. In Proceedings of the 2004 IEEE Aerospace Conference, volume 1339, 2004 .

[15] S. M. Parkes, I. Martin, I. Dunstan, D. Matthews, and V. Silva. Lidar-based gnc for planetary landing: Simulation with pangu. In Proc. DASIA, ESA Pub., volume 532, 2003. 
[16] S. M. Parkes, I. Martin, and I. Milne. Lunar surface simulation - modelling for vision guided lunar landers. In Proc. DASIA, ESA Pub., 1999.

[17] V. Reddy, A. Nathues, L. C. Lucille, H. Sierks, J.-Y. Li, R. Gaskell, T. McCoy, A. W. Beck, S. E. Schröder, C. M. Pieters, K. J. Becker, B. J. Buratti, B. Denevi, D. T. Blewett, U. Christensen, M. J. Gaffey, P. Gutierrez-Marques, M. Hicks, H. U. Keller, T. Maue, S. Mottola, L. A. McFadden, H. Y. McSween, D. Mittlefehldt, D. P. O'Brien, C. Raymond, and C. Russell. Color and albedo heterogenity of vesta from dawn. Science, 336:700-703, 2012.

[18] H. Sierks, H. U. Keller, R. Jaumann, H. Michalik, T. Behnke, F. Bubenhagen, , I. Büttner, U. Carsenty, U. Christensen, R. Enge, B. Fiethe, P. Gutiérrez Marqués, H. Hartwig, H. Krüger, W. Kühne, T. Maue, S. Mottola, A. Nathues, K.-U. Reiche, M. L. Richards, T. Roatsch, S. E. Schröder, I. Szemerey, and M. Tschentscher. The dawn framing camera. Space Science Reviews, 163:263-327, 2011.

[19] T. Whitted. An improved illumination model for shaded display. Comm. ACM, 23(6), 1980 . 